Referencia para citar este artículo: Deambrosio, M., Gutiérrez de Vázquez, M., Arán-Filippetti, V., \& Román, F. (2018). Efectos del Maltrato en la Neurocognición. Un Estudio en Niños Maltratados Institucionalizados y no Institucionalizados. Revista Latinoamericana de Ciencias Sociales, Niñez y Juventud, 16(1), 239-253. doi:10.11600/1692715x.16114

\title{
Efectos del Maltrato en la Neurocognición. Un Estudio en Niños Maltratados Institucionalizados y no Institucionalizados*
}

\author{
Marisa DEAMBrosio \\ Psicóloga Servicio Penitenciario Federal, Argentina. \\ MARISEL GUTIÉRREZ DE VAZZQUEZ ${ }^{* * * *}$ \\ Profesora de la Universidad Adventista del Plata, Argentina. \\ VANESSA ARÁN-FILIPPETTI ***** \\ Investigadora Consejo Nacional de Investigaciones Científicas y Técnicas (Conicet), Argentina. \\ FABIAN ROMÁN $^{* * * * * *}$ \\ Profesor Universidad de la Costa, Colombia y Fundación Hippocampus, Argentina.
}

\section{Artículo recibido en febrero 27 de 2017; artículo aceptado en mayo 16 de 2017 (Eds.)}

- Resumen (analítico): los objetivos del presente estudio fueron: (i) analizar las diferencias en el desempeño de un protocolo neurocognitivo en niños y niñas que experimentaron maltrato en comparación con niños y niñas que no lo sufrieron; $y$ (ii) examinar si existen diferencias entre niños y niñas víctimas de maltrato, institucionalizados y no institucionalizados. Evaluamos a 30 niños y niñas con trauma temprano (11 institucionalizados y 19 no institucionalizados) y 24 controles de 7 a 12 años de edad. Empleamos el WISC IV, el Tomal, el Test de la Mirada, el Test de Metidas de Pata y la Escala Scared. Encontramos diferencias significativas entre los niños y niñas maltratados y no maltratados en todos los dominios analizados. Además, los niños y niñas maltratados institucionalizados obtuvieron un rendimiento inferior en CI respecto de sus pares maltratados no institucionalizados. La experiencia de estrés vivida en forma temprana, facilitaría un neurodesarrollo caracterizado por deficiencias en aspectos cognitivos y emocionales.

Este artículo de investigación científica y tecnológica se deriva de los datos recolectados para la tesis doctoral de la primera autora, realizada entre los meses de marzo de 2011 a mayo de 2013. Para el presente artículo, empleamos un nuevo abordaje con el objetivo de examinar las diferencias neurocognitivas entre niños y niñas maltratados, institucionalizados y no institucionalizados. Nombre de Investigación que dio origen al Artículo: Cognición Social en niños y adolescentes víctimas de violencia. La entidad que avaló el proyecto de investigación fue el Comité Científico de Tesis de la Universidad Maimónides, mediante acta firmada por el Doctor Ricardo Allegri del 25 de agosto del año 2012. Área del Conocimiento: Psicología. Sub-área: Psicología del desarrollo.

** Doctora en Psicología con Orientación en Neurociencia Cognitiva Aplicada, egresada de la Universidad de Maimónides. Psicóloga del Servicio Penitenciario Federal (SPF). Orcid: 0000-0002-5512-5172. Correo electrónico: marisadeambrosio79@gmail.com

*** Licenciada en Psicología y Licenciada en Psicopedagogía, egresada de la Universidad Adventista del Plata. Becaria doctoral del Grupo Vinculado al Centro Interdisciplinario de Investigaciones en Psicología Matemática y Experimental (Ciipme), Consejo Nacional de Investigaciones Científicas y Técnicas (Conicet), profesora de la Universidad Adventista del Plata. Orcid: 0000-0002-5462-0227. Índice H5: 2. Correo electrónico: lic. mariselgutierrez@gmail.com

**** Doctora en Psicología, egresada de la Universidad Católica Argentina. Investigadora del Centro Interdisciplinario de Investigaciones en Psicología Matemática y Experimental (Ciipme), Consejo Nacional de Investigaciones Científicas y Técnicas (Conicet). Orcid: 0000-0002-07535089. Índice H5: 13. Correo electrónico: vanessaaranf@gmail.com

***** Médico psiquiatra. Doctor en Psicología con Orientación en Neurociencia Cognitiva Aplicada, egresado de la Universidad Maimónides. Profesor en la Universidad de la Costa, Barranquilla, Colombia. Arcid: 0000-0001-7741-3602. Indice H5: 3. Correo electrónico: faromanmd@gmail.com 
Palabras clave: neuropsicología, maltrato, institucionalización, niñez, edad (Tesauro de Ciencias Sociales de la Unesco).

\section{Effects of mistreatment on neurocognition: A study on institutionalized and non- institutionalized children who have suffered mistreatment}

- Abstract (analytical): The purpose of this study is: (i) to analyze the differences in the application of a neurocognitive protocol for children that have suffered maltreatment compared to children who have not suffered abuse; and (ii) to examine if there are any differences between institutionalized and non-institutionalized children who have suffered mistreatment. A total of 30 seven-to twelveyear-old children who suffered mistreatment during early childhood (11 institutionalized and 19 noninstitutionalized) and 24 children in a control group were assessed. The WISC IV, the TOMAL, Eyes Test and Faux Pas Recognition Test and the SCARED scale were employed. Significant differences were found between abused and non-abused children in every aspect that was analyzed. Furthermore, abused institutionalized children have shown a dramatically lower performance in their IQ tests compared to non-institutionalized children who were abused. Early stress experiences seem to produce a neurodevelopment characterized by deficiencies in terms of cognitive and emotional aspects.

Key words: Neuropsychology, abuse, institutionalization, childhood, age (Unesco Social Science Thesaurus).

\section{Efeitos do Maltrato na Neurocognição. Um estudo em crianças maltratadas institucionalizadas e não institucionalizadas}

- Resumo (analítico): os objetivos do presente estudo foram (i) analisar as diferenças no desempenho de um protocolo neurocognitivo em crianças que experimentaram maltrato em comparação com crianças que não apresentaram e (ii) examinar se existem diferenças entre crianças maltratadas de forma institucionalizadas e não institucionalizadas. Avaliaram-se 30 crianças com traumas nos primeiros anos de vida (11 institucionalizadas e 19 não institucionalizadas) e 24 com controles de 7 a 12 anos de idade. Foi utilizado o WISC IV, o TOMAL, o Teste do Olhar, o Teste de "metidas de pata" e a Escala SCARED. Encontraram-se diferenças significativas entre as crianças maltratadas e não maltratadas em todos os dominios analisados. Ademais, as crianças maltratadas de forma institucionalizada obtiveram um rendimento inferior em $C I$ em relação ao seus pares maltrados de forma não institucionalizada. A experiencia de stress vivida desde cedo facilitaria o desenvolvimento neurológico caracterizado por deficiências em aspectos cognitivos e emocionais.

Palavras-chave: neuropsicologia, maltrato, institucionalização, infância, idade (Thesaurus de Ciências Socias da Unesco).

\section{-1. Introducción. -2. Metodología. -3. Resultados. -4. Discusión. -Lista de referencias.}

\section{Introducción}

La vivencia de una experiencia traumática, crónica y de carácter interpersonal, como el maltrato por parte del sujeto cuidador, tiene un fuerte impacto en el desarrollo neurobiológico y psicológico del niño o niña. La exposición al maltrato y estrés temprano produce una cascada de eventos neurobiológicos que tienen el potencial de causar cambios duraderos en el desarrollo del cerebro a nivel estructural, funcional y neurohormonal (Cabrera \& Astaiza, 2016; Teicher, Andersen, Polcari, Anderson, Navalta, \& Kim, 2003). El estrés temprano también afecta procesos importantes de desarrollo, incluyendo la neurogénesis, la sobreproducción sináptica, la poda, y la mielinización durante períodos específicos y sensibles (Teicher et al., 2003; Teicher, Tomoda, \& Andersen, 2006).

En los últimos años, la intensidad y la frecuencia con la que ocurren hechos de 
violencia a nivel social y familiar han hecho que esta problemática forme parte de la agenda pública. Tal es así, que el abuso físico, psíquico y la negligencia de la infancia son considerados en el Manual Diagnóstico y Estadístico de Trastornos Mentales (DSM IV) como problemas que son objeto de atención clínica frecuente. Las instituciones educativas, por otra parte, no escapan a las problemáticas de la violencia, encontrándose que gran parte de los niños, niñas y adolescentes que han sido víctimas de maltrato, manifiestan diferentes tipos de dificultades tanto en el funcionamiento cognitivo como en su desarrollo socioemocional.

\subsection{Efectos del maltrato en la neurocognición}

En lo que respecta específicamente al funcionamiento cognitivo, diversos estudios han demostrado que procesos como la inteligencia, las funciones ejecutivas (FE) y la memoria, entre otros, se encuentran afectados por el maltrato infantil. Entre estos, se encuentra por ejemplo el de Cohen-Imach (1999), quien analizó el efecto del maltrato en el funcionamiento intelectual de 30 niños, niñas y adolescentes de 6 a 16 años de edad, que habían sufrido abuso - psíquico, sexual y/o físico-, y halló que un alto porcentaje de la muestra (i.e., $63 \%$ ) se ubicó por debajo de la media esperada para su edad. Consistentemente, Viezel, Freer, Lowell y Castillo (2015) encontraron un menor coeficiente intelectual, valorado a través de la escala de inteligencia de Wechsler para menores, cuarta edición (Wisc IV), en niños y niñas que sufrieron maltrato infantil. Pérez y Widom (1994) también encontraron diferencias significativas entre niños y niñas abusadas y un grupo control, en cuanto al coeficiente intelectual y la habilidad lectora, aun después de controlar la edad, el sexo y el nivel socioeconómico.

Entre las investigaciones que han analizado los efectos del maltrato en las FE, se encuentra, por ejemplo, la de Fay-Stammbach, Hawes y Meredith (2017), quienes examinaron la inhibición, la memoria de trabajo y la flexibilidad cognitiva en 107 niños y niñas de 4 a 5 años de edad que habían sufrido maltrato. Los autores hallaron que la exposición al maltrato predice un porcentaje único de la varianza de las $\mathrm{FE}$, aun después de controlar el lenguaje comprensivo y la depresión materna. Consistentemente, Mothes, Kristensen, GrassiOliveira, Fonseca, Lima-Argimon y Irigaray (2015) examinaron el impacto de diferentes formas de maltrato infantil en el funcionamiento ejecutivo de adolescentes que habían sufrido diferentes tipos de maltrato y un grupo control, y hallaron que los adolescentes que habían sufrido un único tipo de maltrato exhibieron un peor desempeño en tareas que valoran la flexibilidad cognitiva, respecto de aquellos que sufrieron múltiples tipos de maltrato y de un grupo control sin historial de maltrato. Según los autores, es posible que la exposición a un solo tipo de abuso severo sea más perjudicial que la exposición a diferentes tipos de maltrato. Por su parte, Cowell, Cicchetti, Rogosch y Toth (2015) también demostraron el efecto del maltrato infantil en las FE de inhibición y memoria de trabajo en niños y niñas de 3 a 9 años de edad. Finalmente, en una investigación reciente para analizar los efectos del maltrato en diferentes habilidades cognitivas, Hanson, Adluru, Chung, Alexander, Davidson y Pollak (2013) analizaron la planificación, la memoria y el aprendizaje visual y la memoria visuoespacial en 25 niños, niñas y adolescentes que habían sufrido maltrato y un grupo control sin historial de maltrato. Los autores hallaron que los niños, niñas y adolescentes que sufrieron maltrato, presentaron un menor desempeño en todas las tareas empleadas, así como diferencias estructurales en las regiones prefrontales cerebrales, respecto de sus pares no maltratados.

Por otra parte, estudios que han analizado el desarrollo socioemocional en niños y niñas maltratados, también han demostrado que quienes han sufrido maltrato presentan más problemas de conducta (Salzinger, Feldman, Hammer, \& Rosario, 1993; Crittenden, Claussen, \& Sugarman, 1994), mayor cantidad de desórdenes afectivos (Kaufman, 1991) y un funcionamiento social y emocional más pobre (Kelly et al., 2015) respecto de niños $\mathrm{y}$ niñas no maltratados. Específicamente en el área de la cognición social, algunos estudios han encontrado que niños y niñas con trauma temprano tienen alteradas las habilidades discriminativas que influyen en 
el reconocimiento de las emociones faciales (Masten et al., 2008; Pollak \& Kistler, 2002; Pollak \& Tolley-Schell, 2003) y un retraso en el desarrollo de las habilidades de toma de perspectiva social (Burack, Flanagan, Peled, Sutton, Zygmuntowicz, \& Manly, 2006) y de la teoría de la mente (Cicchetti, Rogosch, Maughan, Toth \& Bruce, 2003).

\subsection{Maltrato infantil e instituciona- lización}

Si bien durante toda la vida el ser humano es sensible al estrés, hay ciertos períodos más vulnerables al trauma temprano por lo que los efectos del estrés pueden ser diferenciales según la etapa de desarrollo en que se produjo el daño (Lupien, McEwen, Gunnar, \& Heim, 2009). Por ejemplo, estudios previos han demostrado que el efecto del maltrato sobre el desarrollo cognitivo y socioemocional varía en función del tiempo de inicio y la exposición al mismo -i.e., cronicidad- (Jaffee \& MaikovichFong, 2011; Manly, Kim, Rogosch, \& Cicchetti, 2001). La severidad (Ayoub et al., 2006) y el tipo de maltrato recibido (Crittenden et al., 1994) también son factores que podrían explicar las variaciones en el desempeño cognitivo y conductual de los niños y niñas maltratados.

En este contexto, una variable que podría tener efectos diferenciales sobre el desarrollo neurocognitivo es la institucionalización, es decir, si los niños, niñas y adolescentes que han sufrido maltrato han sido apartados judicialmente o no de su núcleo familiar. Las diferencias podrían deberse a que la institucionalización se asocia a otros factores de riesgo psicosocial como el abandono, la pobreza, la adversidad y la delincuencia familiar (Musitu, Clemente, Escarti, Ruipérez, \& Román, 1990). Estudios que han analizado el desarrollo cognitivo y/o emocional en niños, niñas y adolescentes institucionalizados, han encontrado un menor desarrollo lingüístico (Moreno-Manso, García-Baamonde, \& Blázquez-Alonso, 2010), mayor impulsividad cognitiva (Fernández-Millán, Pérez-Máñez, \& Carrasco-Salmerón, 2002), bajos niveles de autoestima y mayor agresividad (Musitu et al., 1990), y mayores niveles de depresión (González-Fragoso, Ampudia-Rueda, \&
Guevara-Benítez, 2012), respecto de sujetos no institucionalizados. Según nuestro conocimiento, sin embargo, no existen estudios que comparen el desempeño cognitivo y socioemocional entre niños y niñas víctimas de maltrato que se encuentran institucionalizados, y aquellos que también han experimentado maltrato pero que viven en su núcleo familiar. En esta línea se ha demostrado, por ejemplo, el efecto de diferentes sistemas de cuidado, hallándose que niños y niñas que viven en hogares transitorios (i.e., Foster care) obtienen mejores resultados en el tiempo en cuanto al desarrollo socioemocional, respecto de quienes viven en orfanatos (Ahmad et al., 2005).

\subsection{El presente estudio}

Teniendo en cuenta que no hemos encontrado investigaciones que analicen los efectos del maltrato en variables cognitivas y socioemocionales, examinando las diferencias de desempeño en función de la institucionalización o no del niño y/o la niña que ha sufrido maltrato, en el presente estudio analizamos si existen diferencias significativas en un protocolo neurocognitivo en función de la violencia experimentada tempranamente, y comparamos el desempeño de sujetos menores maltratados institucionalizados, frente a otros no institucionalizados. En esta investigación hacemos hincapié en el trauma temprano generado por la violencia familiar, definida como aquellas relaciones intrafamiliares que involucran abuso de poder - emocional, físico y/o sexual — de parte de quien ejerce el maltrato (Ferreira, 2000).

\section{Metodología}

\subsection{Participantes}

Realizamos un estudio ex post facto. La muestra, seleccionada a través de un muestreo no probabilístico intencional, estuvo constituida por 54 niños y niñas. De los 54 individuos participantes, $27(50.0 \%)$ eran mujeres y 27 eran varones. La edad estuvo comprendida en un rango de 7 a 12 años $(\mathrm{M}=9.72, \mathrm{DE}=1.41)$. La muestra estuvo subdividida del siguiente modo: 
1. Grupo Control o muestra no clínica (GC), 24 niños y niñas de 7 a 12 años de edad. Muestra intencional apareada por edad y nivel de instrucción con relación a los grupos de niños maltratados.

2. Grupo no institucionalizado (GNI): 19 niños y niñas escolarizados, de 7 a 12 años de edad, que vivenciaron maltrato y viven con sus familias.

3. Grupo institucionalizado (GI): 11 niños y niñas escolarizados, de 7 a 12 años de edad, que vivenciaron maltrato, fueron apartados de su núcleo familiar y se encuentran institucionalizados.

La muestra que integró los grupos GNI y GI, la seleccionamos mediante observaciones sistemáticas, entrevistas clínicas, documentación existente en las instituciones donde asistían, y mediante la consideración de los indicadores de maltrato aportados por la asociación argentina de prevención de la violencia familiar. Específicamente, escogimos a los niños y niñas del GI de un hogar en el cual viven personas menores que fueron separadas judicialmente de su núcleo familiar por hechos de violencia que las mismas padecieron. Los niños y niñas del GNI vivían con sus familias, pero presentaban indicadores de trauma temprano (véase Tabla 2). Asistían a las mismas escuelas a las que asistían los niños y niñas del grupo control y en las que inclusive asistían algunos de los niños y niñas que pertenecían al grupo de individuos institucionalizados. Excluimos de esta investigación a aquellos niños y niñas que presentaban enfermedades neurológicas, enfermedades sistémicas con afección cerebral y abuso de alcohol o de sustancias.

\subsection{Instrumentos}

Test de Inteligencia para Niños (Wisc IV) de Wechsler (2005)

El Wisc-IV permite valorar la inteligencia en niños, niñas y adolescentes con edades comprendidas entre los 6 y los 16 años. Es un instrumento de administración individual que está conformado por 10 subtests principales y 5 suplementarios. Si bien es posible obtener un Cociente Intelectual Total (CIT) de la persona, también se pueden calcular cuatro índices (i.e., Velocidad de Procesamiento, Memoria
Operativa, Comprensión Verbal y Razonamiento Perceptivo) que brindan información sobre el funcionamiento intelectual en áreas cognitivas específicas. La consistencia interna del test estimada a partir del método de división por mitades - muestra coeficientes de .79 a $.90, \mathrm{y}$ los de estabilidad test-retest, de .77 a .86 .

Test de Memoria y Aprendizaje (Tomal) de Reynolds y Bigler (2001)

El Tomal es una batería que evalúa diferentes aspectos de la memoria y se puede aplicar en edades comprendidas entre 5 y 19 años. Consta de 10 tests principales y cuatro test complementarios, que a su vez se dividen en dos grandes escalas: Memoria verbal y Memoria no verbal. Es posible calcular cuatro índices principales (índice de memoria verbal, índice de memoria no verbal, índice de memoria compuesta, índice de recuerdo demorado e índice de aprendizaje) y cinco índices complementarios (índice de recuerdo asociativo, índice de aprendizaje, índice de recuerdo secuencial, índice de recuerdo libre $\mathrm{y}$ un índice de atención/concentración). La confiabilidad del test, calculada a través de la consistencia interna de los ítems, es de $\alpha 0.86$ a $\alpha 0.96$ para el índice de Memoria verbal, y de $\alpha 0.83$ a $\alpha 0.95$ para el índice de Memoria no verbal.

Test de la Mirada (Baron-Cohen, Wheelwright, Hill, Raste, \& Plumb, 2001)

Es un test para evaluar la capacidad de inferir estados mentales y emocionales a partir de la región de los ojos. La versión para niños y niñas consta de 28 fotografías en blanco y negro, cada una con un par de ojos y cuatro palabras. Por cada fotografía, el niño o niña debe elegir una de esas cuatro opciones dadas, la que a su criterio mejor describa lo que la persona de la foto piensa o siente.

Test de la metida de pata (Baron-Cohen, O'Riordan, Jones, Stone, \& Plaisted, 1999)

Es un test para evaluar la teoría de la mente, diseñado para niños y niñas de edades entre 7 a 11 años. La tarea involucra el reconocimiento de una metida de pata. Consta 
de 20 historias, 10 de ellas con metidas de pata y 10 historias control, sin metidas de pata. Entre las historias se utilizan cuatro preguntas para evaluar la comprensión del niño o niña. La primera pregunta evalúa si el sujeto detecta la metida de pata. La segunda confirma que haya detectado correctamente cuál es esa metida de pata. La tercera asegura que haya comprendido la historia y haya prestado atención, y que la falla en la respuesta no sea por problemas de comprensión o distracción. La última pregunta valora si la metida de pata es una consecuencia de la falsa creencia más que de una acción maliciosa.

Screen for Child Anxiety Related Emotional Disorders (Scared) (Birmaher, Brent, Chiappetta, Bridge, Monga, \& Baugher, 1999)

Es una escala de tipo Likert conformada por 41 ítems que evalúa sintomatología ansiosa en edades comprendidas entre 9 a 18 años de edad. Las opciones de respuesta son: $0=$ nunca o casi nunca, $1=$ algunas veces, $2=$ frecuentemente, casi siempre. La suma de las respuestas da la puntuación total, la cual puede tomar valores de 0 a 82 puntos. La escala reporta buenas propiedades psicométricas. En relación con la consistencia interna, el coeficiente $\alpha$ para el puntaje total es de aproximadamente 0,90. En relación con la validez, el análisis de factores muestra una solución de 5 factores: (1) pánico/somático; (2) ansiedad generalizada; (3) ansiedad de separación; (4) fobia social; y (5) fobia escolar. Cada factor tiene buena consistencia interna, con un coeficiente $\alpha$ con valores que oscilan entre 0,78 a 0,87 . Asimismo, el puntaje de ansiedad total del niño o niña y de cada uno de los 5 factores discriminan significativamente a niños y niñas con trastornos de ansiedad, de niños y niñas con trastornos psiquiátricos no ansiosos $(p<0.005)$. Finalmente, en relación con la sensibilidad y especificidad, un punto de corte de 25 en la escala indica sensibilidad óptima (71\%) y especificidad $(67 \%, 61 \%$ y $71 \%)$ al discriminar entre ansiedad y no ansiedad, ansiedad y depresión, ansiedad y trastornos disruptivos, respectivamente.

\subsection{Aspectos éticos}

En primer lugar, solicitamos autorización a los directivos de las instituciones donde asistían los niños y niñas $\mathrm{y}$, posteriormente, el consentimiento por escrito de los padres o tutores legales para la participación de los niños y niñas en el estudio. A través de una carta explicamos los objetivos de la investigación y el tipo de tareas que desarrollaríamos. Aseguramos que la información dada sería confidencial y aclaramos que la colaboración era voluntaria. Finalmente, antes de proceder a la recolección de datos, obtuvimos el asentimiento del niño o niña para su participación en el estudio.

La presente investigación fue aprobada por el comité del Doctorado en Psicología de la Universidad Maimónides.

\subsection{Procedimientos Estadísticos}

Utilizamos estadísticos descriptivos para determinar los indicadores de maltrato y las características sociodemográficas de la muestra. Para evaluar si había diferencias en cuanto a la edad y a la ansiedad en función de los tres grupos de niños y niñas (institucionalizados, no institucionalizados y grupo control), empleamos Análisis univariado de varianza. Para analizar las diferencias en el desempeño del protocolo neurocognitivo en los niños y niñas que habían experimentado situaciones de maltrato en comparación con aquellos que no habían vivenciado situaciones de maltrato, y entre los niños y niñas institucionalizados y no institucionalizados, con control de la ansiedad, empleamos Análisis Multivariado de Covariancia (Mancova). Efectuamos el procesamiento y el análisis estadístico con el Statistical Package for the Social Sciences (SPSS) versión 20.0.

\section{Resultados}

En la tabla 1 presentamos las características sociodemográficas de la muestra, y en la tabla 2 , los indicadores de maltrato según grupo. 
Tabla 1. Características sociodemográficas de la muestra.

\begin{tabular}{cccc}
\hline & \multicolumn{3}{c}{ Grupo } \\
\cline { 2 - 4 }$N$ & GC & GNI & GI \\
\hline Edad & 24 & 19 & 11 \\
Sexo & $9.29(D E=0.28)^{n s}$ & $10.00(D E=0.32)^{n s}$ & $10.18(D E=0.42)^{n s}$ \\
& $\mathrm{M}=12 / \mathrm{V}=12$ & $\mathrm{M}=8 / \mathrm{V}=11$ & $\mathrm{M}=7 / \mathrm{V}=4$ \\
\hline
\end{tabular}

$n s=$ Diferencias no significativas.

Tabla 2. Indicadores de maltrato y nivel de ansiedad según grupo.

\begin{tabular}{cccc}
\hline & \multicolumn{3}{c}{ Grupo } \\
\cline { 2 - 4 } & GC & GNI & GI \\
\hline Violencia física & No & 17 & 11 \\
Violencia emocional & No & 19 & 11 \\
Violencia sexual & No & No & 1 \\
Índice de maltrato (IM) & 0 & $13.53(3.01)$ & $13.36(2.25)$ \\
Ansiedad** & $15.25(D E=2.69)$ & $28.21(D E=3.02)$ & $30.18(D E=3.97)$ \\
\hline
\end{tabular}

Nota. El IM corresponde a los indicadores de maltrato aportados por la asociación argentina de prevención de la violencia familiar. El índice comprende un rango de 0 a 23 puntos.

Ansiedad **p $<0.01$

\section{Inteligencia}

Dado que no hallamos diferencias significativas según la edad, $F(2,51)=2.175$, $p>0.05$, pero sí en cuanto a la ansiedad entre los tres grupos, $F(2,51)=7.283, p<0.01$, realizamos un Mancova con control de la ansiedad. El Mancova no mostró un efecto principal de la ansiedad, $F$ de Hotelling $(3,48)=$ $0.485, p>0.05, \mathrm{Eta}^{2}$ parcial $=0.029, \beta-1=.141$, pero sí del grupo $F$ de Hotelling $(6,94)=8.144$, $p<0.001, \mathrm{Eta}^{2}$ parcial $=0.342, \beta-1=1.000$ sobre las habilidades intelectuales. Específicamente, estas diferencias las encontramos en todas las variables cognitivas evaluadas, es decir, en la comprensión verbal, $F(2,50)=23.928 ; p$ $<0.001$, Eta $^{2}$ parcial $=0.489, \beta-1=1.000$, el razonamiento perceptivo, $F(2,50)=10.370$; $p<0.001, \mathrm{Eta}^{2}$ parcial $=0.293, \beta-1=0.983 \mathrm{y}$ el coeficiente intelectual general, $F(2,50)=$ 23.958; $p<0.001, \mathrm{Eta}^{2}$ parcial $=0.489, \beta-1=$ 1.000. En la tabla 3 presentamos los valores medios y contrastes post hoc para cada grupo. 
Tabla 3. Desempeño en habilidades intelectuales según los grupos de niños y niñas.

\begin{tabular}{|c|c|c|c|c|c|c|c|c|c|c|}
\hline \multirow{2}{*}{$\begin{array}{l}\text { Variables } \\
\text { cognitivas }\end{array}$} & \multirow{2}{*}{ Tarea } & \multicolumn{2}{|c|}{$\begin{array}{c}\text { GC } \\
(n=24)\end{array}$} & \multicolumn{2}{|c|}{$\begin{array}{c}\text { GNI } \\
(\mathbf{n}=19)\end{array}$} & \multicolumn{2}{|c|}{$\begin{array}{c}\text { GI } \\
(\mathbf{n}=\mathbf{1 1})\end{array}$} & \multirow{2}{*}{ M1-M2 } & \multirow{2}{*}{ M1-M3 } & \multirow{2}{*}{ M2-M3 } \\
\hline & & M1 & DE1 & M2 & DE2 & M3 & DE3 & & & \\
\hline $\begin{array}{l}\text { Comprensión } \\
\text { verbal }\end{array}$ & Wisc IV & 110.76 & 3.13 & 88.79 & 3.37 & 72.61 & 4.44 & $0.000 * * *$ & $0.000 * * *$ & $0.013^{*}$ \\
\hline $\begin{array}{l}\text { Razonamiento } \\
\text { perceptivo }\end{array}$ & Wisc IV & 92.78 & 2.30 & 82.35 & 2.48 & 74.24 & 3.27 & $0.014 *$ & $0.000 * * *$ & 0.140 \\
\hline $\begin{array}{l}\text { Coeficiente } \\
\text { intelectual }\end{array}$ & Wisc IV & 101.89 & 2.31 & 85.72 & 2.48 & 73.73 & 3.27 & $0.000 * * *$ & $0.000 * * *$ & $0.012 *$ \\
\hline
\end{tabular}

$* p<0.05 ; * * * p<0.001$.

Nota: $G C=$ grupo control; $G N I=$ grupo no institucionalizado; $G I=$ grupo institucionalizado.

Memoria

Dado que no hallamos diferencias entre la edad, $\mathrm{F}(2,51)=2.175, p>0.05$ pero sí en cuanto a la ansiedad entre los grupos, $\mathrm{F}(2,51)=7.283$, $p<0.01$, realizamos un Mancova controlando esta última variable. El Mancova no mostró un efecto principal de la ansiedad $F$ de Hotelling $(3,48)=0.346, p>0.05$, Eta $^{2}$ parcial $=0.021$, $\beta-1=0.113$, pero sí del grupo $F$ de Hotelling $(6,94)=3.675, p<0.01, \operatorname{Eta}^{2}$ parcial $=0.190$, $\beta-1=0.948$ sobre la memoria. Específicamente, estas diferencias las encontramos en todas las variables cognitivas evaluadas, es decir, memoria verbal, $F(2,50)=5.126 ; p<0.01$, $\mathrm{Eta}^{2}$ parcial $=0.170, \beta-1=0.801$, memoria no verbal, $F(2,50)=4.023 ; p<.05, \mathrm{Eta}^{2}$ parcial $=$ $0.139, \beta-1=0.692$ y memoria general, $F(2,50)$ $=10.176 ; p<0.001, \mathrm{Eta}^{2}$ parcial $=0.289, \beta-1$ $=0.981$. En la tabla 4 presentamos los valores medios y contrastes post hoc para cada grupo.

Tabla 4. Desempeño en tareas de memoria según los grupos de niños y niñas.

\begin{tabular}{|c|c|c|c|c|c|c|c|c|c|c|}
\hline \multirow{2}{*}{ Variables cognitivas } & \multirow{2}{*}{ Tarea } & \multicolumn{2}{|c|}{$\begin{array}{c}\text { GC } \\
(n=24) \\
\end{array}$} & \multicolumn{2}{|c|}{$\begin{array}{c}\text { GNI } \\
(\mathrm{n}=19)\end{array}$} & \multicolumn{2}{|c|}{$\begin{array}{c}\text { GI } \\
(n=11)\end{array}$} & \multirow{2}{*}{ M1-M2 } & \multirow{2}{*}{ M1-M3 } & \multirow{2}{*}{ M2-M3 } \\
\hline & & M1 & DE1 & M2 & DE2 & M3 & DE3 & & & \\
\hline Memoria verbal & Tomal & 100.16 & 3.05 & 86.78 & 3.28 & 85.72 & 4.33 & $0.018^{*}$ & $0.035^{*}$ & 1.000 \\
\hline Memoria no verbal & Tomal & 101.93 & 2.72 & 92.26 & 2.93 & 89.44 & 3.87 & 0.073 & $0.043 *$ & 1.000 \\
\hline Memoria compuesta & Tomal & 85.43 & 1.97 & 73.57 & 2.12 & 71.82 & 2.79 & $0.001 * * *$ & $0.001 * * *$ & 1.000 \\
\hline
\end{tabular}

$*_{p}<0.05 ; * * * p<0.001$.

Nota: $G C=$ grupo control; $G N I=$ grupo no institucionalizado; $G I=$ grupo institucionalizado.

\section{Cognición social}

Incluimos dentro de esta categoría la capacidad de inferir estados mentales y emocionales (i.e., test de la mirada) y la identificación de metidas de pata (i.e., faux pas). Como no encontramos diferencias según la edad, $\mathrm{F}(2,51)=2.175, p>0.05$, pero sí en cuanto a la ansiedad entre los grupos, F $(2,51)$ $=7.283, p<0.01$, realizamos un Mancova con control de la ansiedad. El Mancova no mostró un efecto principal de la ansiedad, $F$ de Hotelling $(2,49)=1.018, p>0.05, \mathrm{Eta}^{2}$ parcial $=0.040, \beta-1=0.218$, pero sí del grupo 
$F$ de Hotelling $(4,96)=5.465, p<0.001, \mathrm{Eta}^{2}$ parcial $=0.185, \beta-1=0.970$ sobre la cognición social. Específicamente, estas diferencias las encontramos tanto en el reconocimiento de estados mentales, $F(2,50)=6.867 ; p<0.01$, $\mathrm{Eta}^{2}$ parcial $=0.215, \beta-1=0.906$, como en la identificación de metidas de pata, $F(2,50)$ $=8.894 ; p<0.001, \mathrm{Eta}^{2}$ parcial $=0.262, \beta-1$
$=0.964$. Los contrastes post hoc indican que existen diferencias significativas entre el grupo control respecto a los niños y niñas que estaban institucionalizados y los niños que no estaban institucionalizados, a favor del grupo control. En la tabla 5 presentamos los valores medios y contrastes post hoc para cada grupo de niños.

Tabla 5. Desempeño en tareas de cognición social según los grupos de niños y niñas.

\begin{tabular}{|c|c|c|c|c|c|c|c|c|c|c|}
\hline \multirow{2}{*}{ Cognición social } & \multirow{2}{*}{ Indicador } & \multicolumn{2}{|c|}{$\begin{array}{c}\text { GC } \\
(n=24) \\
\end{array}$} & \multicolumn{2}{|c|}{$\begin{array}{c}\text { GNI } \\
(\mathrm{n}=19)\end{array}$} & \multicolumn{2}{|c|}{$\begin{array}{c}\text { GI } \\
(\mathbf{n}=\mathbf{1 1})\end{array}$} & \multirow{2}{*}{ M1-M2 } & \multirow{2}{*}{ M1-M3 } & \multirow{2}{*}{ M12-M3 } \\
\hline & & M1 & DE1 & M2 & DE2 & M3 & DE3 & & & \\
\hline $\begin{array}{l}\text { Reconocimiento } \\
\text { de estados } \\
\text { mentales }\end{array}$ & $\begin{array}{l}\text { Test de la } \\
\text { Mirada }\end{array}$ & 16.46 & 0.85 & 12.20 & 0.91 & 11.75 & 1.20 & $0.006^{* *}$ & $0.010 * *$ & 1.000 \\
\hline $\begin{array}{c}\text { Reconocimiento } \\
\text { de metidas de } \\
\text { pata }\end{array}$ & $\begin{array}{c}\text { Test de } \\
\text { Metidas de } \\
\text { Pata }\end{array}$ & 28.69 & 2.09 & 18.25 & 2.25 & 13.88 & 2.97 & $0.006 * *$ & $0.001 * * *$ & 0.698 \\
\hline
\end{tabular}

\section{Discusión}

En la presente investigación nuestro objetivo fue analizar las diferencias en el desempeño de un protocolo neurocognitivo en niños y niñas que experimentaron situaciones de maltrato, en comparación con niños y niñas no maltratados, así como analizar las diferencias de desempeño entre niños y niñas maltratados institucionalizados y no institucionalizados.

Los resultados nos mostraron, en primer lugar, que los niños y niñas que sufrieron maltrato (institucionalizados y no institucionalizados) obtuvieron rendimientos inferiores en relación con el grupo control, en tareas que valoran la capacidad intelectual y la memoria. En general, si se observan las medias, se ve un mejor rendimiento de los niños y niñas pertenecientes al grupo control, seguido de los niños y niñas que no se encontraban institucionalizados. Así, tanto la inteligencia verbal, como la inteligencia fluida asociada a las regiones prefrontales del cerebro (Gray, Chabris, \& Braver, 2003), como diferentes habilidades de memoria que implican la retención de material verbal y no verbal, se vieron afectadas por el maltrato. Asimismo, encontramos afectadas por el maltrato las dimensiones afectiva y cognitiva de la ToM, con diferencias significativas entre los rendimientos obtenidos por los niños y niñas maltratados institucionalizados y no institucionalizados- $-y$ el grupo control. Específicamente si se observan las medias, se ve un mejor rendimiento del grupo control, seguido de los niños y niñas que no se encontraban institucionalizados. Finalmente, encontramos que los niños y niñas que experimentaron situaciones de maltrato presentaron mayores indicadores de ansiedad respecto de sus pares no maltratados. Estos datos están en línea con estudios que indican que la exposición al estrés en los primeros años de vida se asocia con cambios neurobiológicos en niños y niñas, cambios que pueden ser la base del aumento del riesgo para desarrollar trastornos de ansiedad, depresión y otros trastornos, con posibilidad de persistir en la edad adulta (Heim \& Nemeroff, 2001). En conjunto, nuestros resultados sugieren que las situaciones de violencia vividas en forma temprana impactan negativamente en el desarrollo cognitivo y socioemocional del niño o niña, y confirman estudios previos que también hallaron efectos 
perjudiciales del maltrato sobre las habilidades intelectuales y la memoria (Cohen-Imach, 1999; Moreno-Manso, 2005; Pérez \& Widom, 1994), en componentes específicos de la cognición social (Cicchetti et al., 2003; Masten et al., 2008; Pears \& Fisher, 2005; Pollak \& Kistler, 2002; Pollak \& Tolley-Schell, 2003), así como en el desarrollo de trastornos de la ansiedad (Famularo, Kinscherff, \& Fenton, 1992; Ito, Teicher, Clod, Harper, Magnus, \& Gelbard, 1993; Perry, Pollard, Blakley, Baker \& Vigilante, 1995; Ramírez, 2006).

El estrés es uno de los factores que la literatura científica ha propuesto como mediador de los efectos del maltrato sobre el desarrollo cognitivo y socioemocional. Se ha señalado que el maltrato infantil altera la función del eje hipotalámico-pituitario-adrenal (HPA), los niveles basales de cortisol y la respuesta al estrés, con importantes implicaciones para la salud física y mental (Tarullo \& Gunnar, 2006). El estrés crónico o la exposición prolongada a glucocorticoides y los elevados niveles de catecolaminas afectan perjudicialmente el desarrollo cerebral y cognitivo (Lupien et al., 2009) y el sistema inmune (Watts-English, Fortson, Gibler, Hooper, \& De Bellis, 2006). Específicamente, estudios de neuroimagen han demostrado que el estrés temprano produce cambios estructurales a nivel cortical, en el hipocampo, en la amígdala, en el cuerpo calloso y en estructuras cerebelares (ver Mesa-Gresa \& Moya-Albiol, 2011, para una revisión), y se asocia a las alteraciones que se evidencian en niños y niñas maltratados, en los sistemas neurobiológicos involucrados en su desarrollo cognitivo y en su regulación emocional (De Bellis, 2005).

Por otra parte, la socialización emocional materna (Shipman, Schneider, Fitzgerald, Sims, Swisher, \& Edwards, 2007) y los déficits en la interacción y en las oportunidades de aprendizaje experimentadas por estos niños y niñas (Twardosz \& Lutzker, 2010), han sido propuestos como causas en la explicación del efecto negativo del maltrato sobre el desarrollo cerebral y cognitivo y sobre la regulación emocional de los niños y niñas. No se puede descartar, además, como posible factor causal de las diferencias en las áreas cognitiva y socioemocional, las secuelas neurológicas que pueden generar el maltrato físico y el trauma emocional; el daño estructural cerebral en las áreas cerebrales temporales y frontales ha sido asociado a alteraciones del lenguaje $y$ del aprendizaje, y a déficits de memoria (ver Giménez-Pando, Pérez-Arjona, Dujovny, \& Díaz, 2007, para una revisión). Teniendo en cuenta que en el presente estudio el $93 \%$ de la muestra de niños y niñas maltratados experimentó maltrato físico, y el 100\% maltrato emocional, podríamos hipotetizar que las diferencias de rendimiento en las tareas que valoran el CI verbal y las diferentes habilidades de memoria se deben, entre otros factores, a las posibles secuelas neurológicas del maltrato.

Respecto de los efectos de la institucionalización sobre los procesos cognitivos y socioemocionales, observamos diferencias significativas en cuanto a las habilidades intelectuales. Específicamente, los niños y niñas institucionalizados presentaron un CI inferior al de sus pares maltratados no institucionalizados. En los demás dominios analizados, si bien no hallamos diferencias significativas entre los niños y niñas institucionalizados y no institucionalizados maltratados, sí observamos rendimientos inferiores en todas las tareas de memoria y de cognición social analizadas. Además, los niños y niñas maltratados no institucionalizados obtuvieron un desempeño similar al del grupo control en la tarea de memoria no verbal. Esto sugiere que la institucionalización de niños y niñas maltratados, respecto de su no institucionalización, tendría un efecto negativo aún mayor sobre el desarrollo cognitivo $\mathrm{y}$ socioemocional. Una posible explicación podría encontrarse en las causas mismas que llevan a la institucionalización de un niño o niña. Según Musitu et al. (1990), un sujeto menor institucionalizado es el resultado de la interacción de (a) variables estructurales, como la deprivación social y económica, $\mathrm{y}$ (b) variables familiares como la negligencia física y psíquica, el abuso de sustancias, la violencia, la enfermedad mental o física, la delincuencia y la desaparición de uno o ambos padres. Por lo tanto, el mayor efecto adverso de la institucionalización podría deberse a que la 
causa de la institucionalización del niño o niña englobaría la combinación de diferentes tipos de maltrato como el abandono (Giovannoni, 1971) y la mayor adversidad y violencia familiar (Musitu et al., 1990). Según MesaGresa y Moya-Albiol (2011), el abandono puede provocar mayores efectos adversos que otros tipos de maltrato, en tanto ocasiona que las estructuras cerebrales y las capacidades cognitivas y emocionales no se desarrollen apropiadamente. Así, por ejemplo, autores y autoras que han analizado el efecto de la institucionalización sobre el estilo cognitivo de los niños y niñas, se han preguntado si es la institucionalización o el historial de maltrato lo que influye en la impulsividad cognitiva del individuo (Fernandez-Millán et al., 2002).

En síntesis, nuestros hallazgos sugieren que el maltrato infantil facilitaría un desarrollo neurocognitivo caracterizado por deficiencias en aspectos cognitivos y en el área de la cognición social, y mayores síntomas de ansiedad. Esto sugiere que las condiciones del ambiente en las que un niño o niña vive, durante los primeros años de vida, tienen gran relevancia para su desarrollo neurocognitivo y socioemocional.

Estos resultados tienen importantes implicaciones clínicas, educativas y sociales. En primer lugar, constituyen un importante aporte hacia la comprensión de los factores socioambientales que pueden influir y modular la estructura y la función cerebral. En segundo lugar, permiten repensar políticas públicas e intervenciones tendientes a contrarrestar los efectos negativos a largo plazo del maltrato sobre el desarrollo cognitivo y socioemocional. Diferentes autores y autoras han demostrado que los niños y niñas víctimas de maltrato presentan problemas de adaptación (Wolfe, Scott, Wekerle, \& Pittman, 2001), mayor prevalencia de trastornos psicológicos y conductuales, y menores logros académicos (Lansford, Dodge, Pettit, Bates, Crozier, \& Kaplow, 2002) en la adolescencia y déficits cognitivos en la adultez (Majer, Nater, Lin, Capuron, \& Reeves, 2010). Asimismo, se ha indicado que el maltrato se asocia a un mayor uso de sustancias durante la adolescencia (Moran, Vuchinich, \& Hall, 2004), a mayores índices de delincuencia juvenil
(Lansford, Miller-Johnson, Berlin, Dodge, Bates, \& Pettit, 2007; Smith \& Thornberry, 1995) y a estrés postraumático en la adultez (Marty \& Carvajal, 2005).

Es necesario, por lo tanto, el desarrollo de programas educativos y de formación docente - entre otras acciones posibles_-, que permitan intervenir efectivamente en las redes sociales primarias, y adecuar a estas problemáticas las redes sociales secundarias, para que las situaciones que generan trauma temprano no se conviertan en determinantes ineludibles de un desarrollo humano deficitario. Al respecto, se ha indicado que las intervenciones tempranas que promueven conjuntamente el desarrollo de competencias cognitivas, conductuales y emocionales, serían las más efectivas para reducir la agresión en la niñez y prevenir la violencia en la adolescencia(Escobar, 2005). Por otra parte, las intervenciones que promueven el empleo de estrategias parentales positivas han resultado efectivas para restablecer los niveles de cortisol y la función del eje HPA en niños y niñas que han sufrido maltrato (Fisher, Gunnar, Chamberlain \& Reid, 2000) y el desarrollo de habilidades ejecutivas y de ToM en edad preescolar (Lewis-Morrarty, Dozier, Bernard, Terracciano, \& Moore, 2012).

Si bien en esta investigación arrojamos luz sobre el impacto que generan las situaciones de violencia sobre habilidades neurocognitivas y de cognición social, futuras investigaciones deberían analizar si la presencia de más de un tipo de violencia (violencia familiar vs. violencia social) y/o de diferentes tipos de maltrato (negligencia vs. maltrato físico vs. maltrato emocional) influye diferencialmente en las habilidades neurocognitivas para que los organismos encargados de responder a estas problemáticas puedan dar respuestas efectivas en la práctica. Se ha sugerido que en la recomendación de asistencia y tratamientos para los niños y niñas que han sufrido maltrato, es necesario que los sujetos profesionales reconozcan el perfil de maltrato recibido para ajustar la intervención a las necesidades del niño o niña (Pears, Kim, \& Fisher, 2008). 


\section{Lista de referencias}

Ahmad, A., Qahar, J., Siddiq, A., Majeed, A., Rasheed, J., Jabar, F., \& Von Knorring, A. L. (2005). A 2-year follow-up of orphans' competence, socioemotional problems and post-traumatic stress symptoms in traditional foster care and orphanages in Iraqi Kurdistan. Child: Care, Health and Development, 31(2), 203-215. doi: 10.1111/j.1365-2214.2004.00477.x

Ayoub, C. C., O Connor, E. R. I. N., RappoltSchlichtmann, G., Fischer, K. W., Rogosch, F. A., Toth, S. L., \& Cicchetti, D. (2006). Cognitive and emotional differences in young maltreated children: A translational application of dynamic skill theory. Development and Psychopathology, 18(3), 679. doi: 10.1017/S0954579406060342

Baron-Cohen, S., O'Riordan, M., Stone, V., Jones, R., \& Plaisted, K.(1999). Recognition of faux pas by normally developing children and children with Asperger syndrome or high-functioning autism. Journal of Autism and Developmental Disorders, 29(5), 407418. doi: 10.1023/A:1023035012436

Baron-Cohen, S., Wheelwright, S., Hill, J., Raste, Y., \& Plumb, I. (2001). The "Reading the Mind in the Eyes" test revised version: A study with normal adults, and adults with Asperger syndrome or high-functioning autism. Journal of Child Psychology and Psychiatry, 42(2), 241-251. doi: 10.1111/1469-7610.00715

Birmaher, B., Brent, D. A., Chiappetta, L., Bridge, J., Monga, S., \& Baugher, M. (1999). Psychometric properties of the Screen for Child Anxiety Related Emotional Disorders (Scared): a replication study. Journal of the American Academy of Child \& Adolescent Psychiatry, 38(10), 1230-1236. doi: 10.1097/00004583199910000-00011

Burack, J. A., Flanagan, T., Peled, T., Sutton, H. M., Zygmuntowicz, C., \& Manly, J. T. (2006). Social perspective-taking skills in maltreated children and adolescents. Developmental Psychology, 42(2), 207.

Cabrera, E., \& Astaiza, G. (2016). Secuelas del maltrato infantil.
Revista PsicologiaCientifica.com, 10(11). Recuperado de: http://www. psicologiacientifica.com/secuelas-delmaltrato-infantil

Cicchetti, D., Rogosch, F. A., Maughan, A., Toth, S. L., \& Bruce, J. (2003). False belief understanding in maltreated children. Development and Psychopathology, 15, 1067-1091. doi: 10.1017/ S0954579403000440

Cohen-Imach, S. (1999). Niños maltratados: análisis de los aspectos cognitivos a través del Wisc-III. Revista Iberoamericana de Diagnóstico y Evaluación Psicológica, 1, 53-63.

Cowell, R. A., Cicchetti, D., Rogosch, F. A., \& Toth, S. L. (2015). Childhood maltreatment and its effect on neurocognitive functioning: Timing and chronicity matter. Development and Psychopathology, 27, 521-533. Recuperado de: http://dx.doi. org/10.1017/S0954579415000139

Crittenden, P. M., Claussen, A. H., \& Sugarman, D. B. (1994). Physical and psychological maltreatment in middle childhood and adolescence. Development and Psychopathology, 6(01), 145-164. doi: 10.1017/S0954579400005927

De Bellis, M. D. (2005). The psychobiology of neglect. Child Maltreatment, 10, 150-172. doi: $10.1177 / 1077559505275116$

Escobar, J. H. (2005). La prevención temprana de la violencia: una revisión de programas y modalidades de intervención. Universitas Psychologica, 4(2), 161-177.

Famularo, R., Kinscherff, R., \& Fenton, T. (1992). Psychiatric diagnoses of maltreated children: preliminary findings. Journal of the American Academy of Child \& Adolescent Psychiatry, 31(5), 863-867. doi: 10.1097/00004583-199209000-00013

Fay-Stammbach, T., Hawes, D. J., \& Meredith, P. (2017). Child maltreatment and emotion socialization: Associations with executive function in the preschool years. Child Abuse \& Neglect, 64, 1-12. doi: 10.1016/j. chiabu.2016.12.004

Fernández-Millán, J. M., Pérez-Máñez, D., \& Carrasco-Salmerón,S.(2002).Impulsividad cognitiva en menores maltratados: factores 
influyentes. Universitas Psychologica, I(2), 21-26.

Ferreira, G. (2000). Cartilla para docentes y educadores/as. Buenos Aires: Asociación Argentina de Prevención de la Violencia Familiar.

Fisher, P. A., Gunnar, M. R., Chamberlain, P., \& Reid, J. B. (2000). Preventive intervention for maltreated preschool children: Impact on children's behavior, neuroendocrine activity, and foster parent functioning. J. Am. Acad. Child Adolesc. Psychiat., 39, 1356-1364. doi: 10.1097/00004583200011000-00009

Gray, J. R., Chabris, C. F., \& Braver, T. S. (2003). Neural mechanisms of general fluid intelligence. Nature Neuroscience, 6, 316-322. doi: 10.1038/nn1014

Giménez-Pando, J., Pérez-Arjona, E., Dujovny, M., \& Díaz, F. G. (2007). Secuelas neurológicas del maltrato infantil: revisión bibliográfica. Neurocirugía, 18(2), 95-100.

Giovannoni, J. M. (1971). Parental mistreatment: Perpetrators and victims. Journal of Marriage and the Family, 33, 649-657. doi: 10.2307/349437

González-Fragoso, C., Ampudia-Rueda, A., \& Guevara-Benítez, Y. (2012). Programa de intervención para el desarrollo de habilidades sociales en niños institucionalizados. Acta Colombiana de Psicología, 15(2), 43-52.

Hanson, J. L., Adluru, N., Chung, M. K., Alexander, A. L., Davidson, R. J., \& Pollak, S. D. (2013). Early neglect is associated with alterations in white matter integrity and cognitive functioning. Child Development, 84, 1566-1578. Recuperado de: http://dx.doi.org/10.1111/cdev.12069

Heim, C., \& Nemeroff, C. B. (2001). The role of childhood trauma in the neurobiology of mood and anxiety disorders: preclinical and clinical studies. Biological Psychiatry, 49(12), 1023-1039. doi: 10.1016/S0006-3223(01)01157-X

Ito, Y., Teicher, M. H., Clod, C. A. Harper, D., Magnus, E., \& Gelbard, H. A. (1993). Increased prevalence of electrophysiological abnormalities in children with psyclhological, physical, and sexual abuse. Journal of Neuropsychiatry and Clinical Neurosciences, 5, 401-408. doi: 10.1176/jnp.5.4.401

Jaffee, S. R., \& Maikovich-Fong, A. K. (2011). Effects of chronic maltreatment and maltreatment timing on children's behavior and cognitive abilities. Journal of Child Psychology and Psychiatry, 52(2), 184-194. doi: 10.1111/j.1469-7610.2010.02304.x

Kaufman, J. (1991). Depressive disorders in maltreated children. Journal of the American Academy of Child \& Adolescent Psychiatry, 30(2), 257-265. doi:10.1097/00004583-199103000-00014

Kelly, P. A., Viding, E., Puetz, V. B., Palmer, A. L., Mechelli, A., Pingault, J. B., ... \& McCrory, E. J. (2015). Sex differences in socioemotional functioning, attentional bias, and gray matter volume in maltreated children: A multilevel investigation. Development and Psychopathology, 27, 1591-1609. doi: https://doi.org/10.1017/ S0954579415000966

Lansford, J. E., Dodge, K. A., Pettit, G. S., Bates, J. E., Crozier, J., \& Kaplow, J. (2002). A 12-year prospective study of the long-term effects of early child physical maltreatment on psychological, behavioral, and academic problems in adolescence. Archives of Pediatrics \& Adolescent Medicine, 156(8), 824-830.

Lansford, J. E., Miller-Johnson, S., Berlin, L. J., Dodge, K. A., Bates, J. E., \& Pettit, G. S. (2007). Early physical abuse and laterviolent delinquency: A prospective longitudinal study. Child Maltreatment, 12(3), 233-245. doi:10.1177/1077559507301841

Lewis-Morrarty, E., Dozier, M., Bernard, K., Terracciano, S. M., \& Moore, S. V. (2012). Cognitive flexibility and theory of mind outcomes among foster children: Preschool follow-up results of a randomized clinical trial. Journal of Adolescent Health, 51, S17-S22. doi: 10.1016/j. jadohealth.2012.05.005

Lupien, S. J., McEwen, B. S., Gunnar, M. R., \& Heim, C. (2009). Effects of stress throughout the lifespan on the brain, behaviour and cognition. Nature Reviews 
Neuroscience, 10(6), 434-445. doi: $10.1038 / \mathrm{nrn} 2639$

Majer, M., Nater, U. M., Lin, J. M. S., Capuron, L., \& Reeves, W. C. (2010). Association of childhood trauma with cognitive function in healthy adults: A pilot study. $B M C$ Neurology, 10, 61-71. doi: 10.1186/14712377-10-61

Manly, J. T., Kim, J. E., Rogosch, F. A., \& Cicchetti, D. (2001). Dimensions of child maltreatment and children's adjustment: Contributions of developmental timing and subtype. Development and Psychopathology, 13, 759-782.

Marty, M., \& Carvajal, A. (2005). Maltrato infantil como factor de riesgo de trastorno por estrés postraumático en la adultez. Revista Chilena de Neuro-Psiquiatría, 43(3), 180-187.

Masten, C. L., Guyer, A. E., Hodgdon, H. B., McClure, E. B., Charney, D. S., Ernst, M., ... $\&$ Monk, C. S. (2008). Recognition of facial emotions among maltreated children with high rates of post-traumatic stress disorder. Child Abuse \& Neglect, 32(1), 139-153. doi:10.1016/j.chiabu.2007.09.006

Mesa-Gresa, P., \& Moya-Albiol, L. (2011). Neurobiología del maltrato infantil: el "ciclo de la violencia". Revista de Neurología, 52(8), 489-503.

Moran, P. B., Vuchinich, S., \& Hall, N. K. (2004). Associations between types of maltreatment and substance use during adolescence. Child Abuse \& Neglect, 28(5), 565-574. doi:10.1016/j. chiabu.2003.12.002

Moreno-Manso, J. M. (2005). Estudio sobre las consecuencias del maltrato infantil en el desarrollo del lenguaje. Anales de Psicología, 21(2), 224-230.

Moreno-Manso, J. M., García-Baamonde, M. E., \& Blázquez-Alonso, M. (2010). Desarrollo lingüístico y adaptación escolar en niños en acogimiento residencial. Anales de Psicología, 26(1), 189-196.

Mothes, L., Kristensen, C. H., Grassi-Oliveira, R., Fonseca, R. P., Lima-Argimon, I. I., \& Irigaray, T. Q. (2015). Childhood maltreatment and executive functions in adolescents. Child and Adolescent
Mental Health, 20, 56-62. doi: 10.1111/ camh. 12068

Musitu, G., Clemente, A., Escartí, A., Ruipérez, Á., \& Román, J. M. (1990). Agresión y autoestima en el niño institucionalizado. Quaderns de Psicologia, 10, 231-250.

Pears, K. C., \& Fisher, P. A. (2005). Emotion understanding and theory of mind among maltreated children in foster care: Evidence of deficits. Development and Psychopathology, 17(01), 47-65. doi: 10.1017/S0954579405050030

Pears, K. C., Kim, H. K., \& Fisher, P. A. (2008). Psychosocial and cognitive functioning of children with specific profiles of maltreatment. Child Abuse \& Neglect, 32(10), 958-971. doi: 10.1016/j. chiabu.2007.12.009

Pérez, C. M., \& Widom, C. S. (1994). Childhood victimization and long-term intellectual and academic outcomes. Child Abuse \& Neglect, 18(8), 617-633. doi:10.1016/01452134(94)90012-4

Perry, B. D., Pollard, R. A., Blakley, T. L., Baker, W. L., \& Vigilante, D. (1995). Childhood trauma, the neurobiology of adaptation, and "use-dependent" development of the brain: How "states" become "traits". Infant Mental Health Journal, 16(4), 271-291.

Pollak, S. D., \& Kistler, D. J. (2002). Early experience is associated with the development of categorical representations for facial expressions of emotion. Proceedings of the National Academy of Sciences, 99(13), 9072-9076. doi:10.1073/ pnas. 142165999

Pollak, S. D., \& Tolley-Schell, S. A. (2003). Selective attention to facial emotion in physically abused children. Journal of Abnormal Psychology, 112(3), 323-338. doi: 10.1037/0021-843X.112.3.323

Ramírez, C. (2006). El impacto del maltrato en los niños y las niñas en Colombia. Revista Infancia, Adolescencia y Familia, 1(2), 287-301.

Reynolds, C. R. \& Bigler, E. D. (2001). Tomal: test de memoria y aprendizaje. Madrid: TEA Ediciones.

Salzinger, S., Feldman, R. S., Hammer, M., \& Rosario, M. (1993). The Effects of 
Physical Abuse on Children's Social Relationships. Child Development, 64, 169-187. doi:10.2307/1131444

Shipman, K. L., Schneider, R., Fitzgerald, M. M., Sims, C., Swisher, L., \& Edwards, A. (2007). Maternal Emotion Socialization in Maltreating and Non maltreating Families: Implications for Children's Emotion Regulation. Social Development, 16(2), 268-285. doi: 10.1111/j.14679507.2007.00384.x

Smith, C., \& Thornberry, T. P. (1995). The relationship between childhood maltreatment and adolescent involvement in delinquency. Criminology, 33(4), 451481. doi: 10.1111/j.1745-9125.1995. tb01186.x

Tarullo, A. R., \& Gunnar, M. R. (2006). Child maltreatment and the developing HPA axis. Hormones and behavior, 50(4), 632-639. doi: 10.1016/j.yhbeh.2006.06.010

Twardosz, S., \& Lutzker, J. R. (2010). Child maltreatment and the developing brain: A review of neuroscience perspectives. Aggression and Violent Behavior, 15(1), 59-68. doi: 10.1016/j.avb.2009.08.003

Teicher, M. H., Andersen, S. L., Polcari, A., Anderson, C. M., Navalta, C. P., \& Kim, D. M. (2003). The neurobiological consequences of early stress and childhood maltreatment. Neuroscience \& Biobehavioral Reviews, 27(1), 33-44. doi:10.1016/S0149-7634(03)00007-1

Teicher, M. H., Tomoda, A., \& Andersen, S. L. (2006). Neurobiological consequences of early stress and childhood maltreatment: are results from human and animal studies comparable? Annals of the New York Academy of Sciences, 1071(1), 313-323. doi:10.1196/annals.1364.024

Viezel, K. D., Freer, B. D., Lowell, A., \& Castillo, J. A. (2015). Cognitive abilities of maltreated children. Psychology in the Schools, 52, 92-106. doi: 10.1002/ pits. 21809

Watts-English, T., Fortson, B. L., Gibler, N., Hooper, S. R., \& De Bellis, M. D. (2006). The psychobiology of maltreatment in childhood. Journal of Social Issues,
62(4), 717-736. doi: 10.1111/j.15404560.2006.00484.x

Wechsler, D. (2005). Escala de Inteligencia para niños (WISC IV). Manual de aplicación y Corrección. Madrid: TEA Ediciones.

Wolfe, D. A., Scott, K., Wekerle, C., \& Pittman, A. L. (2001). Child maltreatment: Risk of adjustment problems and dating violence in adolescence. Journal of the American Academy of Child \& Adolescent Psychiatry, 40(3), 282-289. doi: 10.1097/00004583200103000-00007 